\title{
Circumstellar dust shells around long-period variables
}

\section{Molecular layers resulting from shocked carbon-rich atmospheres}

\author{
Ch. Helling ${ }^{1}$ and J. M. Winters ${ }^{1,2,3}$ \\ 1 Institut für Astronomie und Astrophysik, TU Berlin, Sekr. PN 8-1, Hardenbergstraße 36, 10623 Berlin, Germany \\ 2 Max-Planck-Institut für Radioastronomie, Auf dem Hügel 69, 53121 Bonn, Germany \\ 3 DEMIRM, UMR 8540, Observatoire de Paris, 61 Av. de l'Observatoire, 75014 Paris, France
}

Received 5 April 2000 / Accepted 6 October 2000

\begin{abstract}
The existence of warm zones of an enhanced molecular content surrounding the photospheres of AGB stars has been postulated to explain observed molecular absorption/emission components which are in excess of the results obtained from classical hydrostatic model atmospheres. We have analyzed the chemical equilibrium molecular composition of time-dependent models of carbon-rich circumstellar envelopes for various combinations of the stellar parameters and found that groups of molecules appear to be present in different zones with temporally varying temperature and density. The regions of enhanced molecular abundances are situated between the stellar photosphere and the circumstellar dust shell. In the models, these zones are produced by the levitation of the atmospheric gas due to the dissipation of shock waves and/or due to radiation pressure on molecules and dust.
\end{abstract}

Key words. infrared: stars - molecular processes - hydrodynamics - stars: late type - circumstellar matter

\section{Introduction}

High-resolution infrared spectroscopy of pulsating Asymptotic Giant Branch (AGB) stars has revealed a rather detailed and complex picture of the hydrodynamical and thermal conditions in the atmospheres of these objects. In particular, the presence of a dense layer at a temperature of about $1000 \mathrm{~K}$ was found in the transition region between the presumably dust-free stellar photosphere and the cool circumstellar dust shell (CDS), contributing time variable low-excitation absorption lines to the observed (overtone) bands of, e.g., $\mathrm{CO}, \mathrm{OH}$, and $\mathrm{H}_{2} \mathrm{O}$ (Hinkle 1978; Hinkle \& Barnes 1979; Hinkle et al. 1982; Hinkle et al. 1984; Tsuji 1988). Already Hinkle (1978) proposed a pulsation-shock model, which qualitatively explains the occurrence and the dynamical behavior of these layers. Utilizing in particular the low excitation $\mathrm{CO}$ first overtone lines observed from IRC +10216 , Keady et al. (1988) built a semi-empirical model for this object, employing a step-like velocity structure necessary to fit the observed line profiles. This work has been extended by Keady \& Ridgway (1993) to derive radial distributions of $\mathrm{SiO}, \mathrm{SiH}_{4}, \mathrm{C}_{2} \mathrm{H}_{2}, \mathrm{CH}_{4}$, $\mathrm{NH}_{3}$, and $\mathrm{CS}$ in the IRC +10216 circumstellar envelope. Applying an ab-initio hydrodynamic model, Winters et al. (2000) succeeded to model the temporal changes

Send offprint requests to: Ch. Helling, e-mail: chris@astro.physik.tu-berlin.de in the $\mathrm{CO}$ first overtone line profiles, which occur on a time scale of about 6 pulsation periods in the case of IRC +10216 .

The extended atmospheres of cool giants have regained attention in the recent literature due to low-resolution ISO observations in the thermal infrared spectral region $(\lambda \approx 2 \ldots 16 \mu \mathrm{m})$. In many cases these spectra show extra absorption or emission contributions in certain molecular bands which cannot be explained in the framework of classical hydrostatic model atmospheres. In the oxygenrich case, this concerns in particular the molecules $\mathrm{H}_{2} \mathrm{O}$, $\mathrm{CO}_{2}, \mathrm{SO}_{2}$ and $\mathrm{CO}$ (see, e.g. Tsuji et al. 1997; Justtanont et al. 1998; Ryde et al. 1999; Yamamura et al. 1999b) while in carbon-rich stars mainly $\mathrm{C}_{2} \mathrm{H}_{2}, \mathrm{HCN}, \mathrm{CS}$, and $\mathrm{CO}$ seem to be affected (e.g. Aoki et al. 1998, 1999; Yamamura et al. 1998, 1999a; Jørgensen et al. 2000).

To explain these extra absorption/emission contributions, Tsuji et al. (1997) proposed the presence of a warm molecule forming region above the stellar photosphere, which should be related to the molecular layer previously suggested from the high-resolution spectra.

Excess flux in molecular absorption bands has been observed in conjunction with IR emission for which dust is responsible. For example, Yamamura et al. (1998) and Aoki et al. (1999) report on the simultaneous occurrence of the $11 \mu \mathrm{m} \mathrm{SiC}$ dust feature and weakened $\mathrm{C}_{2} \mathrm{H}_{2}$ absorption. In oxygen-rich stars, the simultaneous occurrence of dust and "molecular layers" has been suggested to explain 
an observed correlation between the occurrence of the $\mathrm{CO}_{2} 15 \mu \mathrm{m}$ band and the $13 \mu \mathrm{m}$ dust feature (Justtanont et al. 1998; Ryde et al. 1999). Matsuura et al. (1999) report on a similar correlation between the $\mathrm{H}_{2} \mathrm{O}$ absorption band strength and the mid-IR excess $F_{12} / F_{2.2}$ in early M-type giants.

One attempt to reproduce the excess absorption/emission in the ISO spectra has been to search for a combination of temperature and column density characterizing a plane-parallel layer of prescribed lateral extension relative to the star in order to fit the strength of the observed features. The temperature and density is assumed to be constant within such a layer and one or more of these layers are used to fit the observations (e.g. Yamamura et al. 1998, 1999b). The temperature of the layer determines the shape of the spectral features in LTE in the optically thin case. Also the column density influences the shape of the features if the lines become optically thick. However, it has been shown in Woitke et al. (1999) that the excitation temperatures (vibrational, rotational) decouple from the gas temperature at a certain critical density where non-LTE effects come into play and that the critical density is different for different molecules. Although different molecules can have different excitation temperatures, their radial location might be similar.

Another procedure to interpret this kind of observations is the computation of a synthetic spectrum from a hydrostatic model atmosphere and the artificial addition of some amount of dust in the synthetic spectrum computation in order to achieve the required filling-in of the molecular absorption features by dust emission (e.g. Aoki et al. 1999; Jørgensen et al. 2000). Dynamic effects due to pulsation or radiation pressure on dust, as well as the back-warming of deeper layers resulting from already moderate amounts of dust cannot be taken into account in the framework of such an approach.

The aim of this paper is to investigate the possible formation of warm and dense non-photospheric regions of enhanced molecular abundances in terms of time-dependent hydrodynamic models which consistently include carbon dust formation. Since these models are completely determined by the stellar parameters $T_{0}, L_{0}, M_{\star}, \mathrm{C} / \mathrm{O}$ and by the piston amplitude and period, $\Delta u$ and $P$, respectively, the temperatures and number densities of the molecules as well as their radial distribution are a result of a consistent calculation.

Section 2 contains a description of our modeling approach. In Sect. 3, the results of our model calculations are presented and it is shown that shock waves and pressure inversions can be responsible for the occurrence of molecular layers. The dependence of the molecular column densities on the model parameters is investigated. Section 4 summarizes the results of the paper and Sect. 5 contains our conclusions.

\section{Approach}

Time-dependent models of carbon-rich dynamical atmospheres of AGB stars have been calculated by means of the CHILD ${ }^{1}$-code developed by Fleischer et al. (1992) and Winters et al. (1997) in an updated version of Helling et al. (2000b). The code solves the coupled equation system describing time-dependent hydrodynamics, radiative transfer, chemistry, and dust formation. Dust nucleation, growth and evaporation is treated by the moment method developed by Gail \& Sedlmayr (1988) and Gauger et al. (1990). The interior pulsation of the star is simulated by a sinusoidal variation of the innermost grid point with prescribed period $P$ and velocity amplitude $\Delta u$. The radiative transfer problem is solved in grey approximation applying the Unno-Kondo method (Unno \& Kondo 1976, 1977; Hashimoto 1995). The mean gas opacities have been approximated by the Planck mean gas opacities in Models A and B while a constant gas opacity has been used for Models C, D, E, and F. Table 1 lists the parameters and some resultant quantities of these models. The mean dust extinction efficiency is represented by the Rosseland mean given in Gail \& Sedlmayr (1985) for the Models A, B, C, and F. For Models D and E, tables of the Planck-mean dust opacity derived from the Preibisch et al. (1993) optical constants for amorphous carbon have been used. The number densities of the molecules discussed in Sect. 3 have been computed for a carbon-rich gas of otherwise solar element composition, assuming chemical equilibrium between 149 molecular and ionic species. We mainly discuss those molecules included as line opacity species $\left(\mathrm{CH}, \mathrm{CN}, \mathrm{C}_{2}, \mathrm{C}_{3}, \mathrm{HCN}, \mathrm{C}_{2} \mathrm{H}_{2}, \mathrm{CO}, \mathrm{TiO}, \mathrm{SiO}\right.$, $\left.\mathrm{H}_{2} \mathrm{O}\right)$ in the gas opacity computation. In addition we consider $\mathrm{CS}$ and $\mathrm{OH}$. CS together with $\mathrm{C}_{2} \mathrm{H}_{2}, \mathrm{HCN}$, and $\mathrm{CO}$ has been found by observations to be present in nonphotospheric molecular layers.

\section{Results}

\subsection{Time-dependent, carbon-rich hydrodynamic models}

The atmospheric structure resulting from time-dependent models for dust forming winds can roughly be divided into two different zones (see Figs. 1, 2):

1. The innermost (photospheric) region $\left(\lesssim 1.1 R_{0}\left({ }^{2}\right)\right)$ is characterized by a steep density gradient (e.g., upper panels in Figs. 1 and 2, solid line). The gas elements are periodically hit by shock waves and the gas is compressed behind shocks;

2. The outer region $\left(\gtrsim 1.1 R_{0}\right)$ is characterized by a much shallower density decrease, i.e. a larger scale height. At about $2.2 \ldots 2.4 R_{0}$ dust formation sets in (indicated by the degree of condensation $f_{\mathrm{c}}$, see lower panels, dotted line). Dust growth becomes efficient around $3 R_{0}$

\footnotetext{
${ }^{1}$ Conventional Hydrodynamics Including Lacking Dust.

${ }^{2} R_{0}$ is the stellar radius of the hydrostatic initial model.
} 
Table 1. Model parameters and resulting wind properties ${ }^{(*)}$

\begin{tabular}{|c|c|c|c|c|c|c|c|c|c|c|c|c|c|}
\hline \multirow[b]{2}{*}{ Model } & \multicolumn{8}{|c|}{ model parameter } & \multicolumn{5}{|c|}{ results } \\
\hline & $\begin{array}{l}T_{0} \\
{[\mathrm{~K}]}\end{array}$ & $\begin{array}{c}L_{0} \\
{\left[10^{4} L_{\odot}\right]}\end{array}$ & $\begin{array}{c}M_{\star} \\
{\left[M_{\odot}\right]}\end{array}$ & $\mathrm{C} / \mathrm{O}$ & $\begin{array}{c}\Delta u \\
{\left[\mathrm{~km} \mathrm{~s}^{-1}\right]}\end{array}$ & $\begin{array}{c}P \\
{[\mathrm{~d}]}\end{array}$ & $\begin{array}{c}\chi^{\mathrm{g}} \\
{\left[\mathrm{cm}^{2} / \mathrm{g}\right]}\end{array}$ & $Q^{(* *)}$ & $\begin{array}{c}g \\
{\left[\mathrm{~cm} / \mathrm{s}^{2}\right]}\end{array}$ & $\begin{array}{c}R_{0} \\
{\left[R_{\odot}\right]}\end{array}$ & $\begin{array}{c}\langle\dot{M}\rangle \\
{\left[M_{\odot} / \mathrm{yr}\right]}\end{array}$ & $\begin{array}{c}\left\langle v_{\infty}\right\rangle \\
{[\mathrm{km} / \mathrm{s}]}\end{array}$ & $\begin{array}{c}\left\langle\rho_{\text {dust }} /\right. \\
\left.\rho_{\text {gas }}\right\rangle\end{array}$ \\
\hline A & 2600 & 1 & 1 & 1.8 & 2 & 650 & $\chi_{\text {Planck }}^{\mathrm{g}}$ & (1) & 0.113 & 492 & $3.810^{-7}$ & 18.0 & $1.210^{-3}$ \\
\hline B & 3000 & 1 & 1 & 1.8 & 2 & 650 & $\chi_{\text {Planck }}^{\mathrm{g}}$ & (1) & 0.200 & 369 & $4.610^{-8}$ & 10.2 & $4.910^{-4}$ \\
\hline $\mathrm{C}$ & 2600 & 1 & 1 & 1.8 & 2 & 650 & $210^{-4}$ & $(1)$ & 0.113 & 492 & $9.410^{-6}$ & 31.9 & $4.110^{-3}$ \\
\hline $\mathrm{D}$ & 2600 & 1 & 1 & 1.8 & 4 & 650 & $210^{-4}$ & $(2)$ & 0.113 & 492 & $2.310^{-5}$ & 30.1 & $4.310^{-3}$ \\
\hline $\mathrm{E}$ & 2600 & 0.75 & 1 & 1.8 & 2 & 650 & $210^{-4}$ & $(2)$ & 0.150 & 426 & $7.510^{-6}$ & 30.8 & $4.010^{-3}$ \\
\hline $\mathrm{F}$ & 3000 & 1 & 1 & 1.8 & 2 & 650 & $210^{-4}$ & (1) & 0.200 & 369 & $4.110^{-6}$ & 25.3 & $2.310^{-3}$ \\
\hline
\end{tabular}

(*) The resulting wind properties are arithmetic mean values of the respective time averages taken at 4 radial positions in the outer wind region.

${ }^{(* *)} Q$ is the mean extinction efficiency of the dust grains.

(1) Gail \& Sedlmayr (1985), (2) Preibisch et al. (1993).

and radiation pressure on dust causes an acceleration of the gas by frictional coupling (see the perturbation in the velocity field, e.g., around $2.8 R_{0}$ in the upper diagram of Fig. 1, dotted line). The dust-free region between $\sim 1.1 R_{0}$ and $\sim 2.2 \ldots 2.4 R_{0}$ can be considered as an extended atmosphere.

Due to the large density gradient in the inner region, small amplitude waves, created at the inner boundary, quickly steepen into strong shocks (e.g., upper panels, dotted line). The small amplitude waves at the base of the photosphere (piston position) have velocities comparable with typical micro-turbulence velocities derived from observations (1 . . $5 \mathrm{~km} \mathrm{~s}^{-1}$, e.g., Tsuji 1986).

Shock waves introduce an outward directed, additional force on the gas which causes a levitation of the outer photosphere resulting in densities larger by orders of magnitudes compared to the initial static models (see Figs. 3 and 4 , r.h.s, upper panels). If dust forms, the resulting radiation pressure causes an outward acceleration of the gas due to momentum coupling of the grains to the ambient gas. This mechanism results in the amplification of pre-existing shocks or even in the creation of new, dust induced shock waves. The gas in the post-shock region is compressed which in turn favors dust growth (see density bump (upper panel, dotted line) in Fig. 2 which coincides with the peak of the degree of condensation (lower panel, dotted line)).

The newly-formed dust layer effectively blocks the outgoing radiation and heats the material located further inside (e.g., Fig. 3, r.h.s., top panel, black solid line). This back-warming effect temporarily causes evaporation of already existing dust, prevents the nucleation of new grains and also influences the molecular number densities. This can be observed, e.g., from the r.h.s. panel in Fig. 3 where the locally inwards decreasing number density of molecules

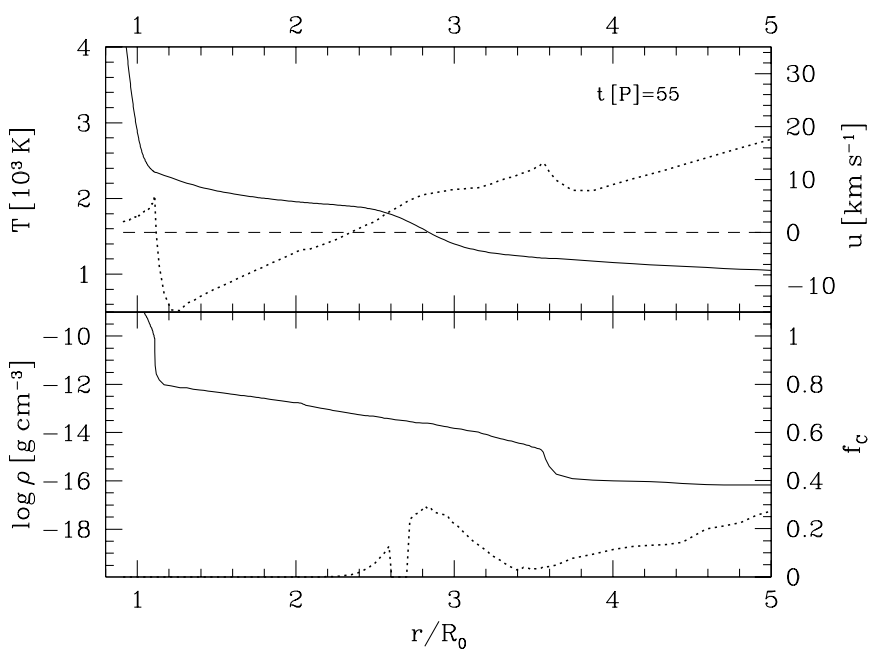

Fig. 1. Radial structure of Model F (constant gas opacity, see Table 1) at one instant of time (full period, i.e. phase $0^{3}$ ). (upper panel: full line - gas temperature $T\left[10^{3} \mathrm{~K}\right]$, dotted line - velocity $u\left[\mathrm{~km} \mathrm{~s}^{-1}\right]$, dashed line - zero velocity line; lower panel: full line - gas density $\rho\left[\mathrm{g} \mathrm{cm}^{-3}\right]$, dotted line - degree of condensation $f_{\mathrm{c}}$ )

like $\mathrm{C}_{2} \mathrm{H}_{2}, \mathrm{HCN}$, and $\mathrm{SiO}$ coincides with the temperature step located around $2.8 R_{0}$.

Besides shock waves, also radiation pressure on molecules may be strong enough to substantially levitate the gas in the dust free atmospheric regions below $r \lesssim 2 R_{0}$. This case is depicted in Fig. 2, where a density inversion occurs around $1.5 R_{0}$ in a model computed with Planck mean gas opacities. Pressure inversions already occur in the relatively cool, static

\footnotetext{
${ }^{3}$ Here, phase 0 corresponds to the rest position of the piston when it is moving outwards with maximum velocity. With this definition, phase 0.25 corresponds to the maximum of the bolometric light curve.
} 


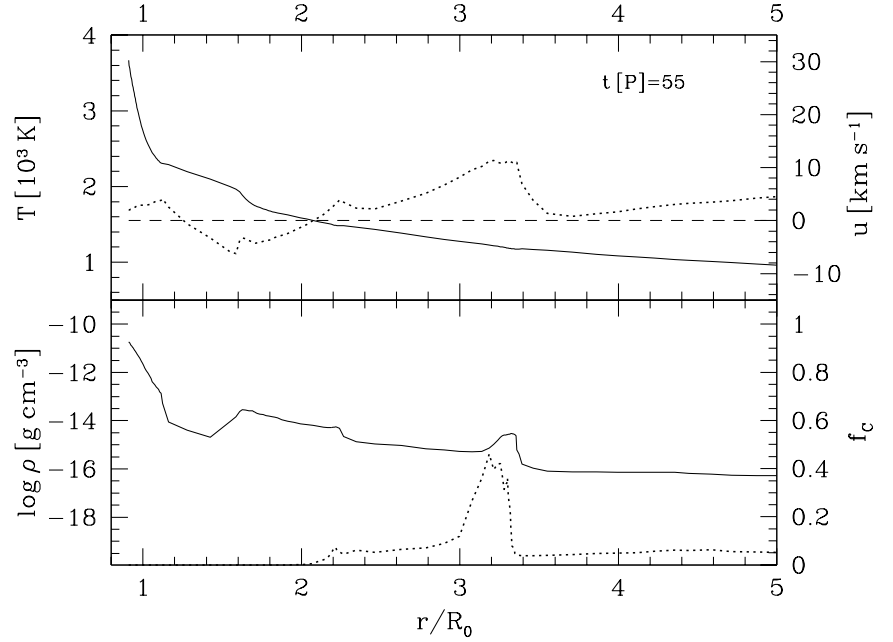

Fig. 2. Same as Fig. 1 for Model B (Planck mean gas opacity, see Table 1)

carbon-rich Planck model (e.g., top panel of Fig. 4, l.h.s.) but not in models computed with Rosseland mean or constant gas opacities (for details see Helling et al. 2000b). If present, these pressure inversions provide another mechanism to enhance the density at small radial distances from the $\operatorname{star}\left(r \approx 1.5 \ldots 2 R_{0}\right)$. In addition, strong molecular opacities also cause a back-warming of the deeper layers. Both effects, the increase of the density and of the temperature, are amplified by shock waves.

\subsection{Molecular layers as result of carbon-rich time-dependent models}

Groups of molecules appear to be present in distinct regions as can be seen from Fig. 3 (Model F) and Fig. 4 (Model B) which depict - as an example - the radial structures at one instant of time of two models computed with different gas opacities. The inner zone around $1 R_{0}$ already occurs in the hydrostatic initial models. A second zone of enhanced molecular abundances forms around $2 R_{0}$ in the time-dependent models caused by the very slowly decreasing or even re-increasing density in combination with an enhanced gas temperature compared to the static case. The molecules most strongly affected in this region are $\mathrm{CH}, \mathrm{CN}, \mathrm{C}_{2}, \mathrm{C}_{3}, \mathrm{C}_{2} \mathrm{H}_{2}$, and $\mathrm{HCN}$.

The compression of the gas by shock waves causes a subsequent efficient dust growth at $\approx 3 R_{0}$ (Figs. 1 and 2 ). Simultaneously, a third local maximum of the number densities occurs for $\mathrm{C}_{2} \mathrm{H}_{2}$ and $\mathrm{HCN}$. Also $\mathrm{SiO}, \mathrm{TiO}$, and $\mathrm{H}_{2} \mathrm{O}$ show such a third maximum in the case of large gas opacities (e.g. Model B, Fig. 4) but it appears to be only the second maximum in the case of small gas opacities (e.g. Model F, Fig. 3). The carbon-bearing di-atomic molecules and also $\mathrm{C}_{3}$ are virtually unaffected in this third zone.

The peak in the molecular number densities around $3 R_{0}$ coincides with the region of enhanced density in the zone of efficient dust growth, produced by the compression of the gas in the wake of the dust-accelerated shock.
A pressure inversion caused by the gas opacity provides an additional levitation mechanism in the Planck models. It causes locally increasing molecular densities in the static model AND in the time-dependent model around 1.6 $R_{0}$ (see Fig. 4). The second maximum of the number densities of $\mathrm{CH}, \mathrm{CN}, \mathrm{C}_{2}, \mathrm{CS}, \mathrm{C}_{3}, \mathrm{C}_{2} \mathrm{H}_{2}$ and $\mathrm{HCN}$ coincides with the location of the pressure inverted region in these models. The local density enhancement caused by the pressure inversion leads to an increased optical depth in this region, resulting in a back-warming of the layers between $r \approx 1.2 \ldots 1.6 R_{0}$ which increases the gas temperature by $\approx 200 \mathrm{~K}$ (Figs. 2,4 ). Shock waves are damped and reflected in the pressure inversion region (see Fig. 2, for details see also Helling et al. 2000b) and as a result the outer atmosphere is less levitated in such models. Due to the smaller overall gas density, the molecular number densities in the Planck models are generally smaller than in models computed with the (small) constant gas opacity. In the constant opacity models (Figs. 1, 3), the dust opacity causes an efficient back-warming with a temperature increase of $\approx 400 \mathrm{~K}$, which reduces the molecular number densities inside of the dust layer (located at $r \approx 3 R_{0}$ in Figs. 1 and 3).

Governed by the local hydrodynamic situation, the molecules which appear to be present in different zones of enhanced molecular densities move with different velocities. The innermost zone oscillates with respect to its rest position and moves with velocities between $-10 \mathrm{~km} \mathrm{~s}^{-1}$ and $6 \mathrm{kms}^{-1}$. The velocity in the second zone around $2 R_{0}$ reaches larger positive velocities than the gas in the first zone but it still can reach also large negative values of $\approx-14 \mathrm{~km} \mathrm{~s}^{-1}$, indicating a high-speed in-fall. The velocity of the matter in this second zone is usually smaller in the Planck models due to the damping of the shocks in the pressure inversion region. The hydrodynamic velocity in the third zone of enhanced molecular densities is largely influenced by radiation pressure on dust. It is always positive in this zone and can reach values up to $\approx 15 \mathrm{~km} \mathrm{~s}^{-1}$ behind the shock front.

Summarizing this section we find, that three distinct zones (I, II, III) of enhanced molecular abundances can be identified in the model atmosphere, which form in the inner shell region $r \lesssim 4 R_{0}$ and which are influenced by different physical mechanisms. Since we assume chemical equilibrium, the properties of these zones of enhanced molecular abundances are determined by the local gas temperature and the local gas pressure alone, which in turn depend on the hydrodynamic structure of the model induced by the interaction of stellar pulsation with the gas- and dust opacities. By applying the law of mass action for calculating the molecular composition of the gas, in particular the polyatomic molecules are affected by the varying local density in the wind-driving zone. The following table should only be considered as a rough characterization of the three inner zones of enhanced molecular content. The numbers given are representative for the models considered in this paper and the values for an individual model lie inside the intervals given. 

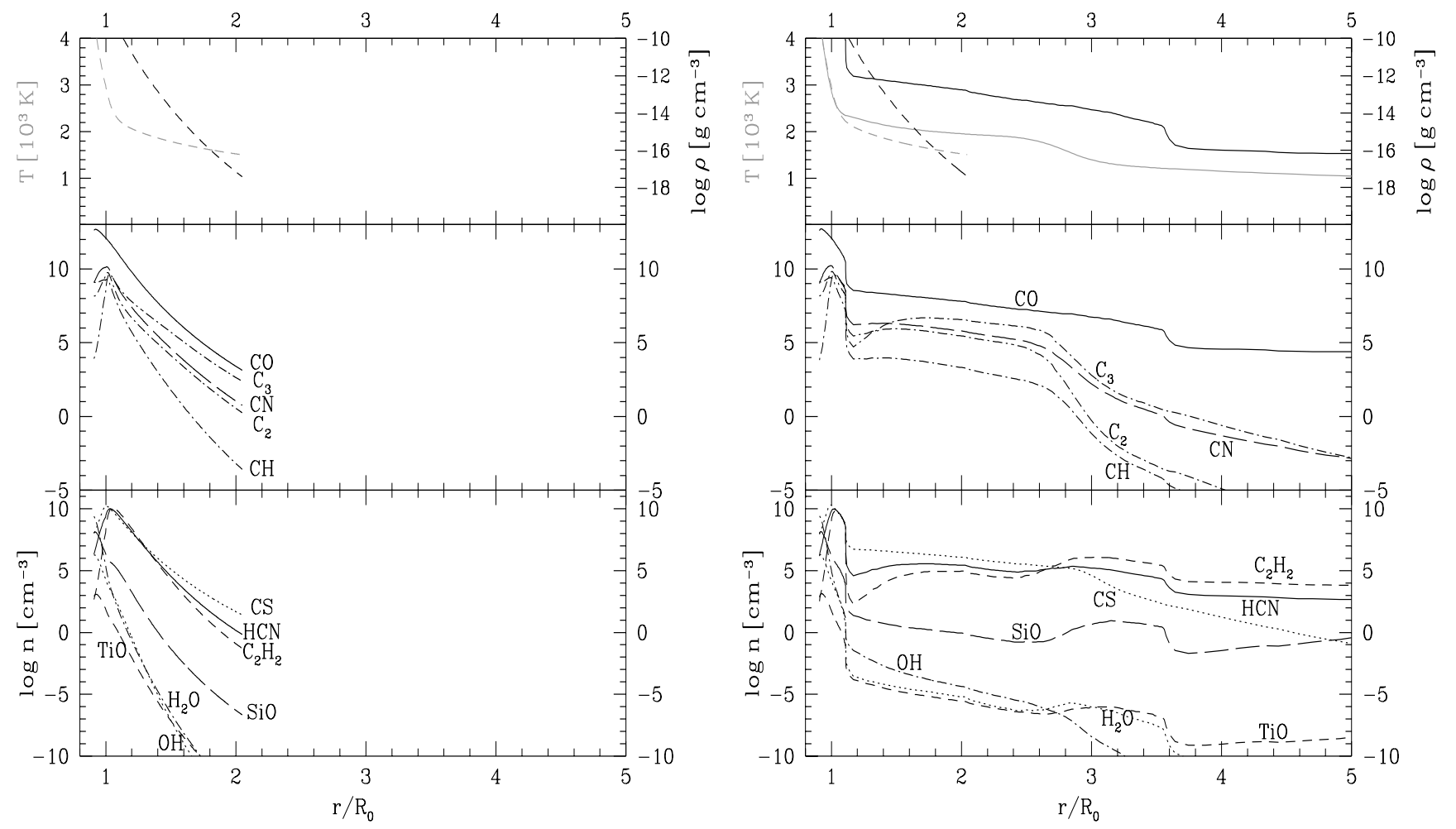

Fig. 3. Molecular number densities $n$ for Model F (small constant gas opacity) in the hydrostatic case (l.h.s.) and for the hydrodynamic case (r.h.s.). The instant of time depicted is the same as in Fig. 1. Upper panel: temperature (dashed blackstatic, full black-dynamic), gas density (dashed grey-static, full grey-dynamic)
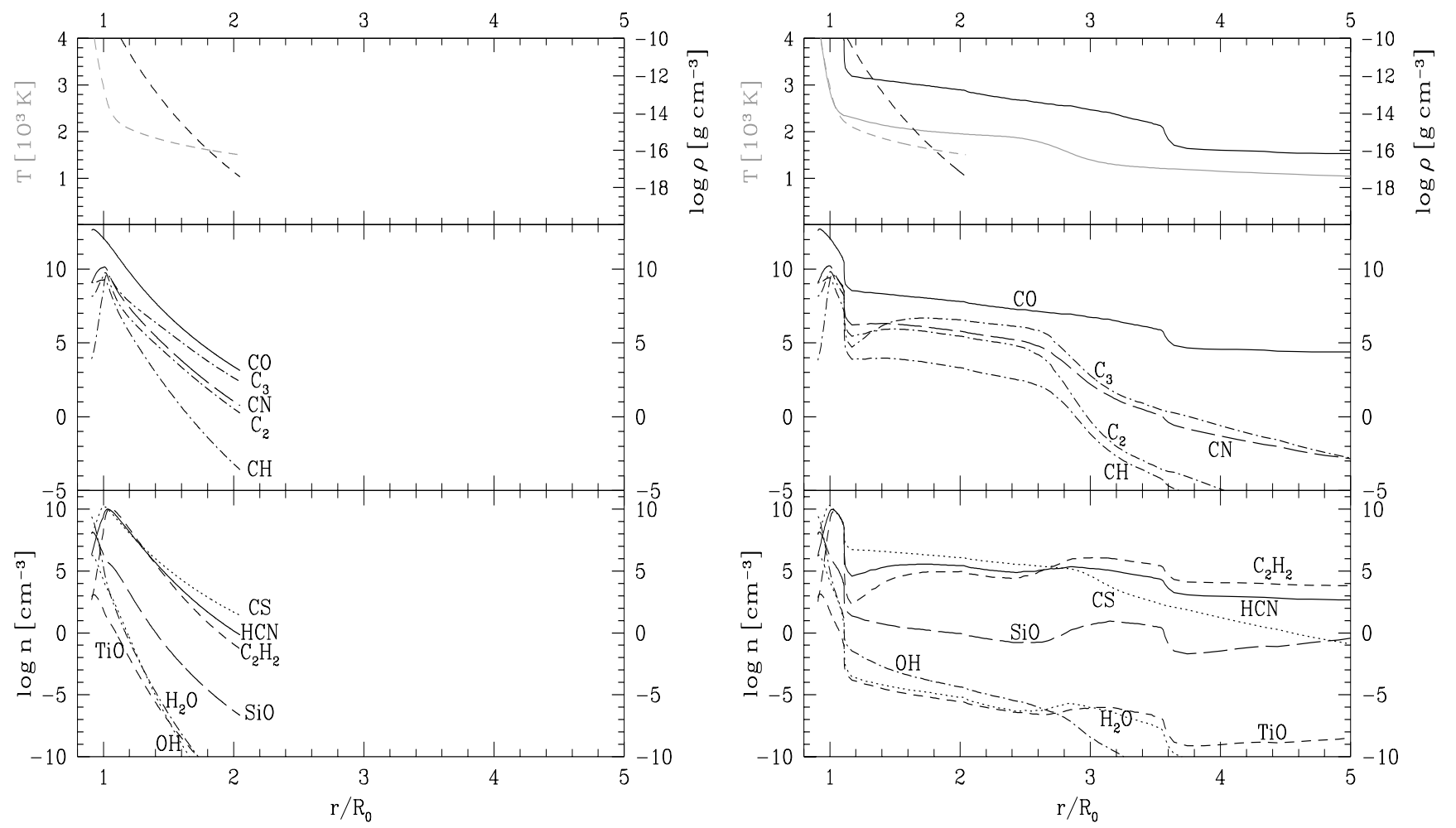

Fig. 4. Same as Fig. 3 for Model B (Planck mean gas opacity) 


\section{Zone I}

(first max., physical conditions:

photosphere)

$$
\text { periodic shocks }
$$

characteristic values:

$v \approx-10 \mathrm{~km} \mathrm{~s}^{-1} \ldots 6 \mathrm{~km} \mathrm{~s}^{-1}$

$T \approx 3500 \mathrm{~K} \ldots 1500 \mathrm{~K}$

$r \approx 0.9 R_{0} \ldots 1.2 R_{0}$

affects

all molecules

\section{Zone II physical conditions:}

(second max., levitation by shock wave dissipation or "extended radiation pressure on molecules,

atmosphere") back-warming by dust or molecules (intermediate layer)

characteristic values:

$v \approx-8 \mathrm{~km} \mathrm{~s}^{-1} \ldots 15 \mathrm{~km} \mathrm{~s}^{-1}$

$T \approx 2000 \mathrm{~K} \ldots 1000 \mathrm{~K}$

$r \approx 1.5 R_{0} \ldots 2.5 R_{0}$

affects

carbon-bearing molecules:

$\mathrm{CO}, \mathrm{CN}, \mathrm{CH}, \mathrm{CS}, \mathrm{C}_{2}, \mathrm{C}_{3}, \mathrm{C}_{2} \mathrm{H}_{2}, \mathrm{HCN}$ and $\mathrm{SiO}\left(\mathrm{TiO}, \mathrm{H}_{2} \mathrm{O}\right.$, OH in negligible amounts)

\section{Zone III}

(third max., physical conditions:

inner CDS)

radiation pressure on dust

(dust-determined layer)

\section{characteristic values:}

$v \gtrsim 7 \mathrm{~km} \mathrm{~s}^{-1}$

$T \lesssim 1500 \mathrm{~K}$

$r \approx 3 R_{0} \ldots 4 R_{0}$

affects

$\mathrm{CO}$, polyatomic molecules, and $\mathrm{SiO}$

( $\mathrm{TiO}, \mathrm{H}_{2} \mathrm{O}$ in negligible amounts).

\subsection{Definition of an inner boundary of the extended atmosphere}

In order to separate the molecular content of the extended atmosphere from the contribution of the photosphere, we define a column density $\mathcal{N}_{y}^{\text {col }}$ and a total number density $N_{y}^{\text {tot }}$ of molecular species $y$ in the region above the photosphere by:

$\mathcal{N}_{y}^{\text {col }}=\int_{\mathrm{r}_{1}}^{\mathrm{r}_{2}} n \mathrm{~d} r$,

$N_{y}^{\mathrm{tot}}=4 \pi \int_{\mathrm{r}_{1}}^{\mathrm{r}_{2}} n r^{2} \mathrm{~d} r$.

The outer integration boundary $r_{2}$ corresponds to the outermost radial position of the model whereas the determination of the inner integration boundary $r_{1}$ poses a crucial problem since the photosphere is perturbed by propagating shocks. The aim is to determine an inner boundary which allows to deduce the effect of shock waves on the non-photospheric region. Simply stated, we wish to exclude the photospheric contribution to the number densities in the time-dependent models as well as in the initial static models. However, the identification of e.g., a

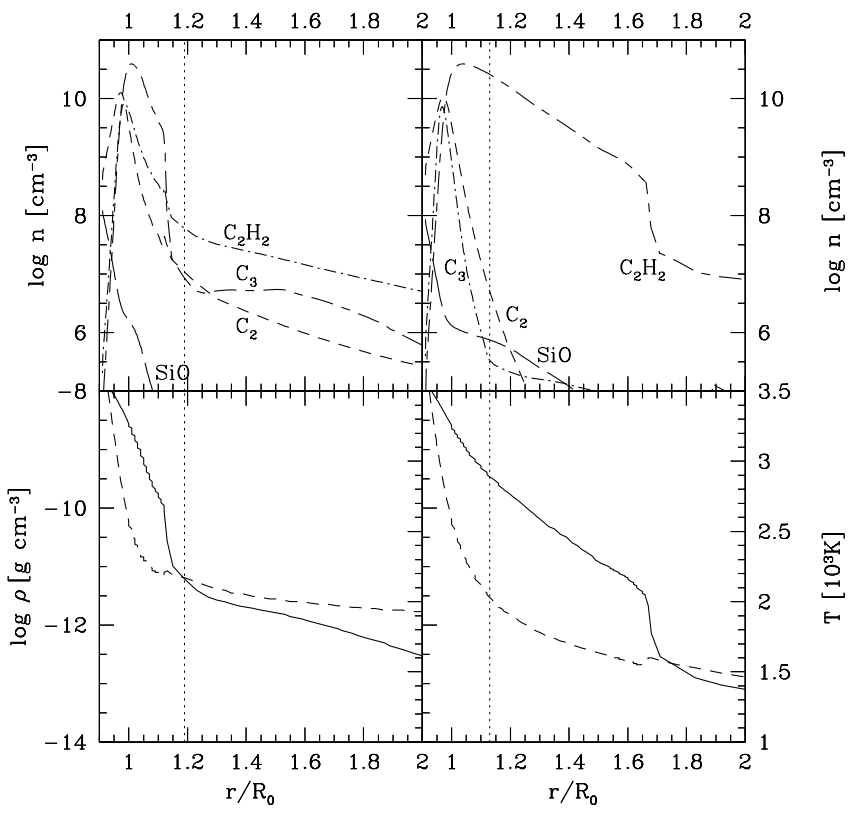

Fig. 5. Variation of the inner integration boundary $r_{1}$ for Model E between phase 0 (l.h.s) and phase 0.5 (r.h.s.). (upper panel: molecular particle densities $n\left[\mathrm{~cm}^{-3}\right]$, (vertical) dotted line - location of $r_{1}$ (see text); lower panel: solid line gas density $\log \left(\rho\left[\mathrm{g} \mathrm{cm}^{-3}\right]\right)$, dashed line - gas temperature $T$ $\left.\left[10^{3} \mathrm{~K}\right]\right)$

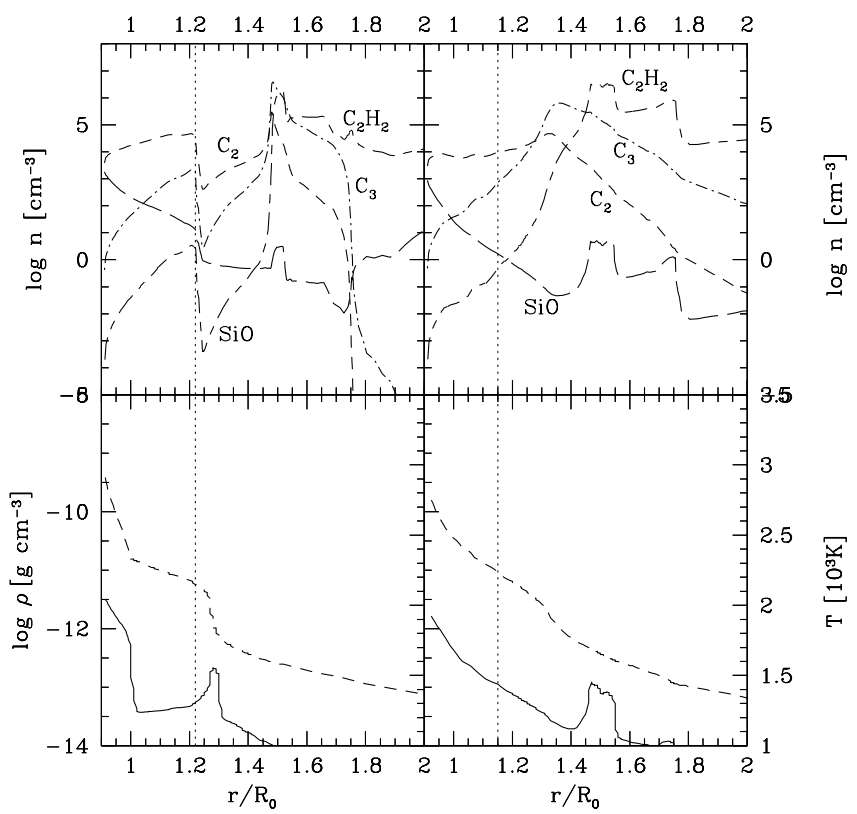

Fig. 6. Same as Fig. 5 for Model C

unique radial position, corresponding to a local minimum of the molecular number densities, as transition between the photosphere and the extended atmosphere is rather difficult. Since the photosphere is periodically hit by shock waves, such a minimum point does not necessarily exist at every instant of time (e.g. CS in Fig. 3) and the choice of a truncation criterion equally applicable to all models and all molecules is not obvious. 
Figures 5 and 6 (lower panels) depict the gas temperature and the density in the inner region of Models $\mathrm{E}$ and $\mathrm{C}$, respectively, at phase 0 and 0.5 . One observes that at phase 0.5 the density step in Model $\mathrm{E}$ and the pressure inversion in Model $\mathrm{C}$ have moved outward causing a smooth transition from the photosphere to the extended atmosphere. Since we are mainly interested in separating the dynamically influenced part of the atmosphere from the photospheric contribution - which corresponds more or less to the static model - we base our criterion for the inner integration boundary on the local density scale height $H_{\rho}^{0}(r)$ calculated from the actual thermodynamic and hydrodynamic conditions of the gas. The evaluation of the local density scale height from the actual thermodynamic conditions in the dynamic model, which makes it dependent on $r$ and $t$, results in a moving inner boundary for the extended atmosphere which follows the hydrodynamic behavior of the model to some extent.

We define the inner integration boundary $r_{1}$ by:

$r_{1} \equiv\left\{\min (r) \mid r-\left(R_{0}+4 H_{\rho}^{0}(r)\right) \geq 0\right\}$

with $\quad H_{\rho}^{0}(r)=\frac{k T(r) r^{2}}{\mu m_{\mathrm{H}} G M_{\star}}$.

$R_{0}$ is the stellar radius of the static initial model, $k$ the Boltzmann constant, $r$ the radial position, $T(r)$ the local gas temperature, $\mu$ the mean molecular weight, $m_{\mathrm{H}}$ the mass of the hydrogen atom, $G$ the gravitation constant, and $M_{\star}$ the stellar mass. By its definition Eq. (3), the inner integration boundary $r_{1}$ is located at the minimum radial distance from the star, which fulfills the condition $r \geq R_{0}+4 H_{\rho}^{0}(r)\left(^{4}\right)$.

In Figs. 5 and 6 the inner boundary $r_{1}$ is indicated by the vertical dotted lines at about $1.2 R_{0}$ and one observes that the value of $r_{1}$ depends on the model considered and that it moves depending on the phase of pulsation. The area to the right of this line is the integration region considered when deriving the results presented in the following sections.

\subsection{Dependence of the column densities on the phase of pulsation}

Figure 7 displays the column densities $\mathcal{N}_{y}^{\text {col }}$ (outside of $r_{1}$ ) resulting from the time-dependent models A...F at 5 phase points during one pulsation cycle. The influence of the phase variation (i.e. the piston position) on the column densities amounts to a maximum of about 3-4 orders of magnitude between phase 0.0 (rest position of the piston) and 0.25 (maximum outwards position of the piston, light maximum) in the models with constant gas opacity. These models are characterized by the formation of a large amount of dust. The variation of the column densities with phase in these models is much larger than in the Planck

\footnotetext{
${ }^{4}$ Our choice of $4 H_{\rho}$ is not compelling, one could also use 3 or $5 H_{\rho}$. The point is to reflect the fact, that the photosphere is geometrically extended by "a few scale heights".
}

models (open and filled circles) where only a variation of about 0.5 dex occurs since the shock waves are damped in the pressure inversion region and dust formation is much less efficient due to the reduced overall density.

The column densities at phase 0 and 1 are very similar in all models considered here, since the $T-\rho$ structures are very similar at both phases in the dust-free region. The hotter models (filled symbols, Models B, F) produce smaller column densities at these phases than the cooler models (open symbols, models $\mathrm{A}$ and $\mathrm{C}$ ). The comparison of the Models A and B and the Models $\mathrm{C}$ and $\mathrm{F}$ shows further that the column densities calculated for the hotter Models (B, F) are more sensitive to the phase variation than are the column densities derived for the cooler Models (A, C).

\subsection{Dependence of the column densities on the stellar parameters}

In order to illustrate the influence of the stellar parameters on the column densities more clearly, the results for the constant opacity models $\mathrm{C}, \mathrm{D}, \mathrm{E}$, and $\mathrm{F}$ are again displayed in Fig. 8. The parameters of Models D, E, and $\mathrm{F}$ are varied with respect to Model $\mathrm{C}$ which serves as a standard model ${ }^{5}$. We kept the $\mathrm{C} / \mathrm{O}$ ratio constant. Since the value of $\mathrm{C} / \mathrm{O}=1.8$ is rather high, we may cover the upper limit of the temperature- and density sensitivity of the polyatomic molecules.

The largest effect in comparison to Model C (open square) occurs for Model $\mathrm{F}$ (filled square) which has a higher stellar temperature $\left(T_{0}=3000 \mathrm{~K}\right)$. An increase of the piston amplitude by $2 \mathrm{~km} \mathrm{~s}^{-1}$ (Model D, tripod symbol) affects the column densities more strongly than a decrease of the stellar luminosity by $2500 L_{\odot}$ (Model E, triangle) for most of the molecules. The molecules most sensitive to the stellar parameters are the polyatomic molecules $\mathrm{HCN}$ and $\mathrm{C}_{2} \mathrm{H}_{2}$.

The variation with phase of the column densities of $\mathrm{CH}, \mathrm{CN}, \mathrm{C}_{2}$, and $\mathrm{C}_{3}$ is much less pronounced than the phase variations of $\mathrm{C}_{2} \mathrm{H}_{2}, \mathrm{HCN}$, CS, and CO. This indicates that the main fraction of $\mathrm{CH}, \mathrm{CN}, \mathrm{C}_{2}$, and $\mathrm{C}_{3}$ is located at small radial distances where the dust does not yet directly influence the dynamical structure. A comparison of the r.h.s. of Fig. 3 with Fig. 1 and the r.h.s. of Fig. 4 with Fig. 2 shows that the main non-photospheric amount of $\mathrm{C}_{2} \mathrm{H}_{2}$ and $\mathrm{HCN}$ and a considerable amount of $\mathrm{CO}$ and $\mathrm{CS}$ is located at sites where dust formation takes place. Since the radiation pressure on dust is strongest at phase 0.25 , it possesses the largest influence on the column densities of the molecules $\mathrm{C}_{2} \mathrm{H}_{2}, \mathrm{HCN}, \mathrm{CS}$, and $\mathrm{CO}$ at this phase. In contrast, $\mathrm{CH}, \mathrm{CN}, \mathrm{C}_{2}$, and $\mathrm{C}_{3}$ can only be influenced by dust back-warming but not by levitation due to dust induced shock waves. Therefore, the variation of the column densities of these molecules is much less pronounced.

\footnotetext{
${ }^{5}$ Model C corresponds to Model E in Fleischer et al. (1992).
} 


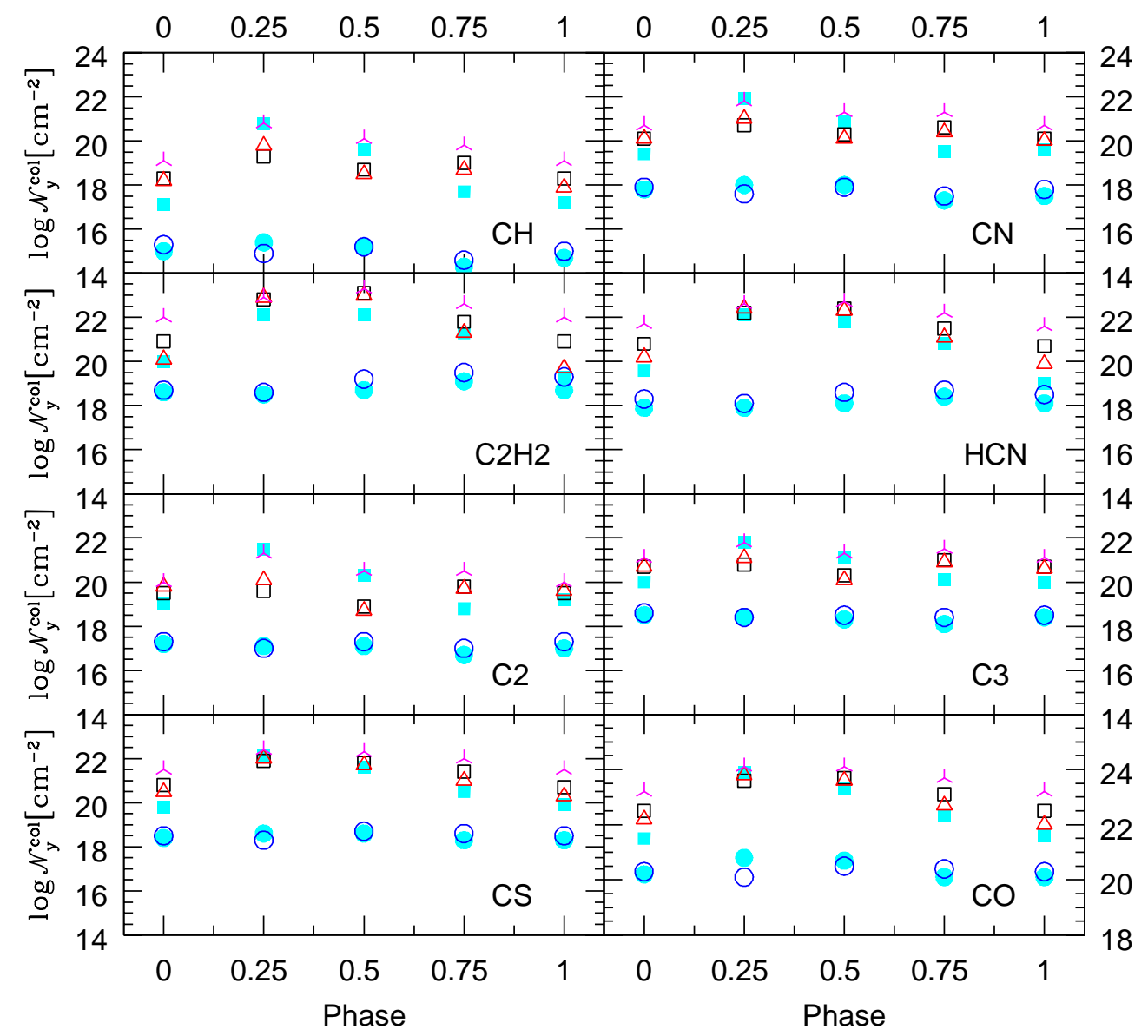

Fig. 7. Temporal variation of the non-photospheric molecular column densities $\mathcal{N}_{y}^{\text {col }}\left[\mathrm{cm}^{-2}\right]$ for the models listed in Table 1 . The circles denote Planck models (open circle - Model A, filled circles - Model B), all other models are computed with constant gas opacities (open squares - Model C, filled squares - Model F, triangles - Model E, tripod symbol - Model D). Note that $\mathcal{N}_{y}^{\text {col }}$ covers a range of 10 magnitudes for all molecules, for CO the scale has been shifted by 2 magnitudes

Within the parameter range investigated, the dependence of the molecular column densities on the stellar parameters are smaller than the variations of the column densities with phase of the stellar pulsation for all molecules.

In Table 2, the arithmetic phase-mean values of the column densities of $\mathrm{CH}, \mathrm{CN}, \mathrm{C}_{2} \mathrm{H}_{2}, \mathrm{HCN}, \mathrm{C}_{2}, \mathrm{C}_{3}, \mathrm{CO}$, $\mathrm{TiO}, \mathrm{SiO}, \mathrm{H}_{2} \mathrm{O}, \mathrm{CS}$, and $\mathrm{OH}$ are compared with the column densities in the static models and Table 3 gives the same for the total particle numbers.

The mean column densities $\left\langle\mathcal{N}_{y}^{\text {col }}\right\rangle$ and mean total particle numbers $\left\langle N_{y}^{\text {tot }}\right\rangle$ are larger in the dynamic case than in the static case for all molecules in all models considered here. One exception occurs for the column density of $\mathrm{CH}$ in Model A, where the phase-mean dynamic value and the static value are equal. From Figs. 7 and 8 one may observe that at some phases the dynamic column density $\mathcal{N}_{y}^{\text {col }}$ can even be smaller than the static value but the mean values $\left\langle\mathcal{N}_{y}^{\text {col }}\right\rangle$ are always larger.

The largest increase of the mean values of column density and total particle number with respect to the corresponding static model is found for Model D, which has the largest amplitude of the interior pulsation
$\left(\Delta u=4 \mathrm{~km} \mathrm{~s}^{-1}\right)$. In this model, the column density of CO increases by about a factor of 25 with respect to the static case. The largest increase among the remaining carbonbearing molecules occurs for $\mathrm{C}_{2}$ (factor $\approx 2600$ ), the smallest for $\mathrm{C}_{2} \mathrm{H}_{2}$ (factor 11). TiO shows the largest increase compared to the static model (factor of $\approx 9800$ ) among all molecules considered (compare Table 2) but the actual number densities are very small.

One may further note that the treatment of the molecular opacity is more important for the amount of molecules present in the models than the variation in the stellar parameters (Fig. 7). The column densities are largest in the constant gas opacity models since the overall gas density is higher than in the Planck models with the same stellar parameters (see Höfner et al. 1998; Helling et al. 2000b).

The Models C, D, E, and F (constant gas opacity) generally show a stronger increase, with respect to the static models, of the mean column density and total particle number for all molecules, than Models A and B (Planck mean gas opacity). For example, the column density of CO increases only by a factor of 2 compared to the static value, $\mathrm{C}_{2} \mathrm{H}_{2}$ increases by a factor of 3 , and $\mathrm{C}_{2}$ increases by only $19 \%$ in Model A. By contrast, the column 
Table 2. Arithmetic phase-mean values of the non-photospheric column densities $\left\langle\mathcal{N}_{y}^{\mathrm{col}}\right\rangle\left[\mathrm{cm}^{-2}\right]$ derived from the time-dependent models listed in Table 1 (marked with A...F) and for the column densities of the corresponding static initial models (marked with $\mathrm{A}^{\mathrm{i}} \ldots \mathrm{F}^{\mathrm{i}}$ )

\begin{tabular}{|c|c|c|c|c|c|c|c|c|c|c|c|}
\hline & $\mathrm{A}^{\mathrm{i}}$ & $\mathrm{A}$ & $\mathrm{B}^{\mathrm{i}}$ & B & $\mathrm{C}^{\mathrm{i}}=\mathrm{D}^{\mathrm{i}}$ & $\mathrm{C}$ & D & $\mathrm{E}^{\mathrm{i}}$ & $\mathrm{E}$ & $\mathrm{F}^{\mathrm{i}}$ & $\mathrm{F}$ \\
\hline $\mathrm{CH}$ & $1.210^{15}$ & $1.210^{15}$ & $4.710^{14}$ & $1.210^{15}$ & $6.510^{16}$ & $7.810^{18}$ & $1.710^{20}$ & $1.310^{16}$ & $1.510^{19}$ & $2.810^{18}$ & $1.310^{20}$ \\
\hline $\mathrm{CN}$ & $5.310^{17}$ & $5.910^{17}$ & $3.610^{17}$ & $6.310^{17}$ & $7.210^{18}$ & $2.710^{20}$ & $2.310^{21}$ & $2.010^{18}$ & $3.210^{20}$ & $1.110^{20}$ & $1.810^{21}$ \\
\hline $\mathrm{C}_{2} \mathrm{H}_{2}$ & $5.210^{18}$ & $1.510^{19}$ & $5.610^{17}$ & $5.910^{18}$ & $6.010^{21}$ & $3.910^{22}$ & $6.810^{22}$ & $1.110^{21}$ & $3.610^{22}$ & $1.310^{21}$ & $5.410^{21}$ \\
\hline $\mathrm{HCN}$ & $2.410^{18}$ & $3.110^{18}$ & $4.210^{17}$ & $1.310^{18}$ & $1.110^{21}$ & $9.010^{21}$ & $2.310^{22}$ & $2.210^{20}$ & $9.310^{21}$ & $7.010^{20}$ & $3.910^{21}$ \\
\hline$\overline{\mathrm{C}_{2}}$ & $1.310^{17}$ & $1.610^{17}$ & $6.010^{16}$ & $1.110^{17}$ & $1.610^{17}$ & $3.510^{19}$ & $5.610^{20}$ & $5.210^{16}$ & $5.710^{19}$ & $2.210^{19}$ & $6.810^{20}$ \\
\hline $\mathrm{C}_{3}$ & $2.910^{18}$ & $3.110^{18}$ & $1.610^{18}$ & $2.310^{18}$ & $1.210^{19}$ & $5.710^{20}$ & $2.810^{21}$ & $4.810^{18}$ & $6.210^{20}$ & $2.610^{20}$ & $1.610^{21}$ \\
\hline $\mathrm{CO}$ & $1.710^{20}$ & $2.210^{20}$ & $1.210^{20}$ & $3.110^{20}$ & $2.610^{22}$ & $2.210^{23}$ & $6.710^{23}$ & $5.610^{21}$ & $2.210^{23}$ & $2.710^{22}$ & $2.010^{23}$ \\
\hline $\mathrm{TiO}$ & $7.510^{6}$ & $4.110^{12}$ & $8.010^{6}$ & $6.110^{13}$ & $1.710^{11}$ & $6.410^{14}$ & $1.710^{15}$ & $1.410^{10}$ & $8.010^{14}$ & $7.110^{10}$ & $4.210^{14}$ \\
\hline $\mathrm{SiO}$ & $6.510^{12}$ & $6.410^{15}$ & $3.110^{12}$ & $3.810^{16}$ & $9.410^{16}$ & $1.810^{18}$ & $3.410^{18}$ & $9.610^{15}$ & $1.710^{18}$ & $1.210^{16}$ & $4.610^{17}$ \\
\hline $\mathrm{H}_{2} \mathrm{O}$ & $2.810^{7}$ & $2.310^{9}$ & $4.210^{6}$ & $1.810^{8}$ & $5.010^{12}$ & $3.310^{14}$ & $1.810^{15}$ & $3.010^{11}$ & $5.610^{14}$ & $4.910^{12}$ & $3.210^{14}$ \\
\hline $\mathrm{CS}$ & $3.210^{18}$ & $3.510^{18}$ & $1.810^{18}$ & $2.910^{18}$ & $3.810^{20}$ & $3.610^{21}$ & $1.210^{22}$ & $9.010^{19}$ & $3.310^{21}$ & $5.210^{20}$ & $3.410^{21}$ \\
\hline$\overline{\mathrm{OH}}$ & $8.010^{7}$ & $4.010^{8}$ & $1.910^{9}$ & $1.510^{11}$ & $1.110^{11}$ & $2.410^{13}$ & $7.510^{14}$ & $8.610^{9}$ & $6.710^{13}$ & $2.110^{12}$ & $6.410^{14}$ \\
\hline
\end{tabular}

Table 3. Same as Table 2 for the non-photospheric total particle numbers $\left\langle N_{y}^{\text {tot }}\right\rangle$

\begin{tabular}{|c|c|c|c|c|c|c|c|c|c|c|c|}
\hline & $\mathrm{A}^{\mathrm{i}}$ & $\mathrm{A}$ & $B^{i}$ & B & $\mathrm{C}^{\mathrm{i}}=\mathrm{D}^{\mathrm{i}}$ & $\mathrm{C}$ & $\mathrm{D}$ & $\mathrm{E}^{\mathrm{i}}$ & $\mathrm{E}$ & $\mathrm{F}^{\mathrm{i}}$ & $\mathrm{F}$ \\
\hline$\overline{\mathrm{CH}}$ & $3.010^{43}$ & $3.010^{43}$ & $7.910^{42}$ & $2.010^{43}$ & $1.410^{45}$ & $1.710^{47}$ & $3.510^{48}$ & $2.210^{44}$ & $2.010^{47}$ & $3.210^{46}$ & $1.710^{48}$ \\
\hline $\mathrm{CN}$ & $1.310^{46}$ & $1.610^{46}$ & $6.010^{45}$ & $1.110^{46}$ & $1.610^{47}$ & $6.310^{48}$ & $5.810^{49}$ & $3.310^{46}$ & $5.710^{48}$ & $1.310^{48}$ & $2.210^{49}$ \\
\hline $\mathrm{C}_{2} \mathrm{H}_{2}$ & $1.510^{47}$ & $9.110^{47}$ & $1.410^{46}$ & $7.610^{47}$ & $1.310^{50}$ & $8.010^{50}$ & $2.010^{51}$ & $1.910^{49}$ & $5.810^{50}$ & $1.510^{49}$ & $8.310^{49}$ \\
\hline $\mathrm{HCN}$ & $6.410^{46}$ & $1.410^{47}$ & $1.010^{46}$ & $6.110^{46}$ & $2.410^{49}$ & $2.010^{50}$ & $5.910^{50}$ & $3.710^{48}$ & $1.510^{50}$ & $8.110^{48}$ & $5.010^{49}$ \\
\hline $\mathrm{C}_{2}$ & $3.210^{45}$ & $4.410^{45}$ & $1.110^{45}$ & $2.310^{45}$ & $3.410^{45}$ & $9.510^{47}$ & $1.210^{49}$ & $8.910^{44}$ & $1.110^{48}$ & $2.610^{47}$ & $8.510^{48}$ \\
\hline $\mathrm{C}_{3}$ & $7.310^{46}$ & $8.410^{46}$ & $3.710^{46}$ & $5.710^{46}$ & $2.710^{47}$ & $1.510^{49}$ & $8.010^{49}$ & $8.410^{46}$ & $1.210^{49}$ & $3.110^{48}$ & $2.010^{49}$ \\
\hline $\mathrm{CO}$ & $4.510^{48}$ & $1.010^{49}$ & $2.010^{48}$ & $9.810^{48}$ & $5.610^{50}$ & $5.110^{51}$ & $1.810^{52}$ & $9.510^{49}$ & $3.710^{51}$ & $3.210^{50}$ & $2.310^{51}$ \\
\hline $\mathrm{TiO}$ & $1.910^{35}$ & $3.210^{43}$ & $1.010^{35}$ & $1.510^{44}$ & $3.610^{39}$ & $1.610^{45}$ & $3.810^{45}$ & $2.310^{38}$ & $1.310^{45}$ & $8.010^{38}$ & $8.910^{44}$ \\
\hline $\mathrm{SiO}$ & $1.710^{41}$ & $6.410^{46}$ & $4.410^{40}$ & $8.810^{46}$ & $2.010^{45}$ & $2.010^{48}$ & $7.910^{48}$ & $1.610^{44}$ & $2.510^{48}$ & $1.310^{44}$ & $6.710^{47}$ \\
\hline $\mathrm{H}_{2} \mathrm{O}$ & $7.310^{35}$ & $5.610^{40}$ & $6.110^{34}$ & $7.310^{38}$ & $1.010^{41}$ & $1.210^{44}$ & $1.810^{45}$ & $5.010^{39}$ & $3.410^{44}$ & $5.510^{40}$ & $5.210^{44}$ \\
\hline CS & $8.410^{46}$ & $1.110^{47}$ & $3.210^{46}$ & $5.810^{46}$ & $8.210^{48}$ & $8.210^{49}$ & $2.910^{50}$ & $1.610^{48}$ & $5.410^{49}$ & $6.010^{48}$ & $4.310^{49}$ \\
\hline $\mathrm{OH}$ & $1.810^{36}$ & $8.210^{36}$ & $2.310^{37}$ & $1.510^{39}$ & $2.310^{39}$ & $4.810^{41}$ & $1.510^{43}$ & $1.410^{38}$ & $1.010^{42}$ & $2.310^{40}$ & $6.410^{42}$ \\
\hline
\end{tabular}

density of $\mathrm{TiO}$ increases by a factor of $\approx 510^{5}$ compared to the static situation in Model $\mathrm{A}^{\mathrm{i}}$. The TiO column density and its total particle number are most sensitive to the increased density caused by the shock waves since $\mathrm{TiO}$ is practically not present in the carbon-rich static case (the same applies to $\mathrm{SiO}$ and $\mathrm{H}_{2} \mathrm{O}$ ).

\section{Discussion}

Investigations of time-dependent carbon-rich models have been performed regarding the chemical equilibrium molecular composition of the gas. We have demonstrated that these models offer an explanation for the presence of molecules in remarkable amounts in non-photospheric regions due to the levitation of the atmosphere by shock waves or/and pressure inversions. Groups of molecules appear to be present in different zones which are characterized by different physical conditions like e.g. periodic pulsations, dust formation, back-warming.

The comparison of the column densities $\left\langle\mathcal{N}_{y}^{\text {col }}\right\rangle$ listed in Table 2 with the values determined by integrating the molecular number densities over the whole radial range of the model including the photosphere shows that the main fractions of $\mathrm{CH}$ and $\mathrm{CO}$ are clearly of photospheric origin whereas a considerable fraction of $\mathrm{C}_{2} \mathrm{H}_{2}, \mathrm{HCN}$, and $\mathrm{C}_{3}$ can be found in a region outside of the photosphere. The exact fraction depends on the model considered and is given in Table 4.

The main contribution to the single molecular column densities often originates from one non-photospheric zone only, whose properties vary in time and depend on the stellar parameters. E.g., for Models B and F one can approximately identify the main contributions to originate from $r \approx 1.5 \ldots 3 R_{0}$ for $\mathrm{CN}, \mathrm{CH}, \mathrm{C}_{2}, \mathrm{C}_{3}, \mathrm{OH}$ and 


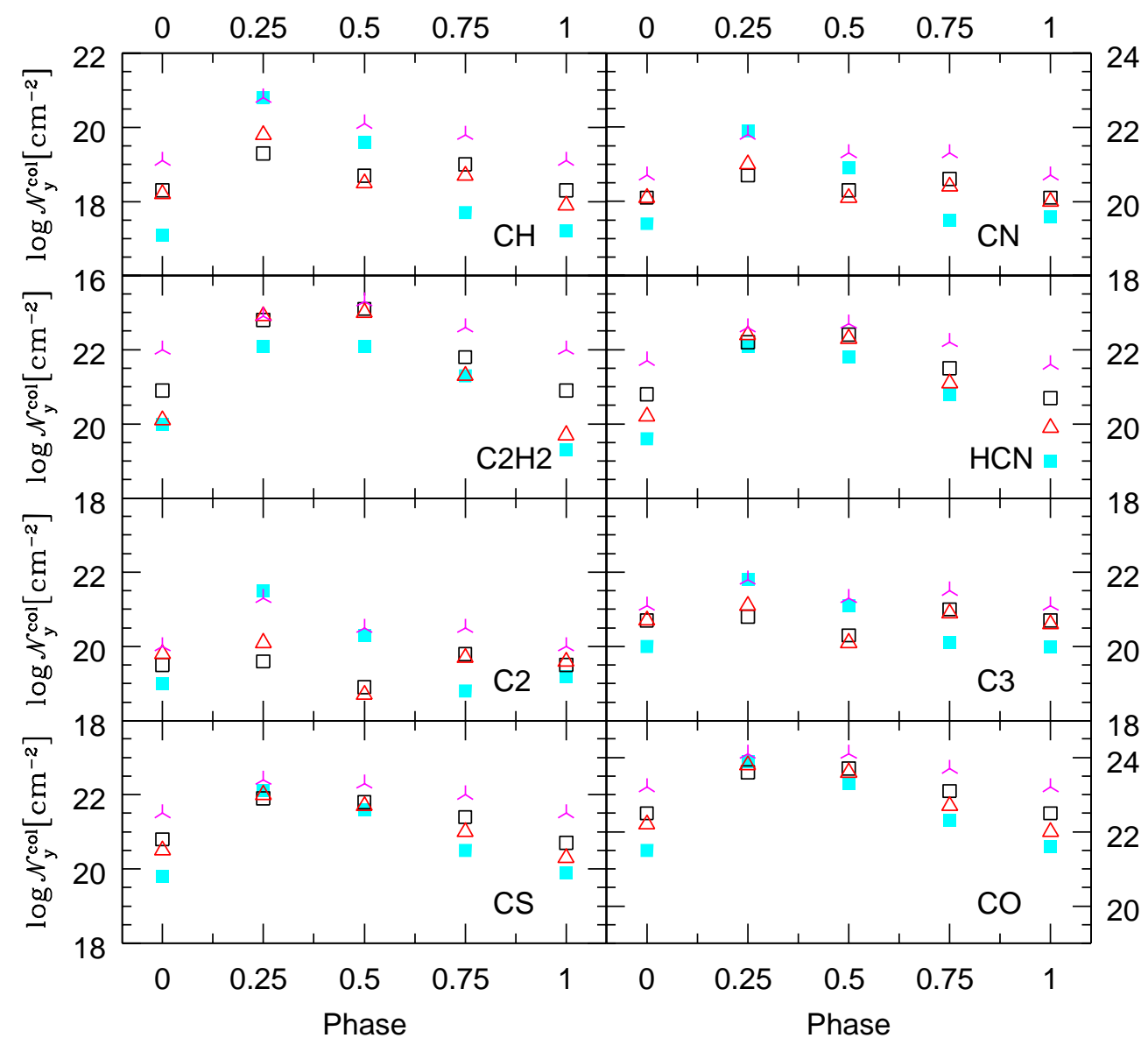

Fig. 8. Same as Fig. 7 for the models computed with a constant gas opacity. Open squares - Model C, filled squares - Model F, triangles - Model E, tripod symbol - Model D. Note that $\mathcal{N}_{y}^{\text {col }}$ spans a range of 6 magnitudes for all molecules and that the scales for $\mathrm{CO}$ and $\mathrm{CH}$ are shifted

from $r \approx 3 \ldots 3.6 R_{0}$ for $\mathrm{C}_{2} \mathrm{H}_{2}$ and $\mathrm{HCN}$ (compare also Table 4).

The only molecule for which column densities in the extended atmosphere have been determined from ISO observations of carbon-rich stars is $\mathrm{C}_{2} \mathrm{H}_{2}$ (Yamamura et al. 1998, 1999a). Applying a simple plane parallel model of a molecular layer to fit observed low-resolution ISO spectra in the $14 \mu \mathrm{m}$ region, Yamamura et al. (1999a) derived a typical value of $\mathcal{N}_{\mathrm{C}_{2} \mathrm{H}_{2}}^{\mathrm{col}}=510^{18} \mathrm{~cm}^{-2}$ and an excitation temperature of $T_{\mathrm{ex}}=1400 \mathrm{~K}$.

One could try to relate the derived temperatures to radial distances. However, one has to be aware that non-LTE effects might come into play. Woitke et al. (1999) show for an oxygen-rich environment that for $\mathrm{CO}, \mathrm{CO}_{2}, \mathrm{SO}_{2}$, and $\mathrm{H}_{2} \mathrm{O}$ the vibrational and rotational excitation temperatures decouple from the gas temperature at certain critical densities which are different for different molecules. Since a similar behavior has to be expected also for the carbon-rich molecules, the determination of the molecule's radial location from a typical excitation temperature derived from the shape of the observed features might not be straight-forward.

From our time-dependent models, we obtain $\mathrm{C}_{2} \mathrm{H}_{2}$ particle densities which are comparable with the values derived from observations: The maximum $\mathrm{C}_{2} \mathrm{H}_{2}$ particle densities occur at $3 \ldots 3.6 R_{0}$ which is inside the dust nucleation zone. This radial position corresponds to an equilibrium gas temperature range of $1400 \ldots 1200 \mathrm{~K}$ for $T_{0}=$ $3000 \mathrm{~K}$ and to a range of $1200 \ldots 1000 \mathrm{~K}$ for $T_{0}=2600 \mathrm{~K}$. This temperature range compares well with the values given by Yamamura et al. $(1998,1999 a)$ for $\mathrm{C}_{2} \mathrm{H}_{2}$. Similar temperatures $(T>1000 \mathrm{~K})$ have also been estimated by Aoki et al. (1999) and are assigned to a warm envelope by these authors. In order to reproduce the wings of the $3 \mu \mathrm{m}$ $\mathrm{C}_{2} \mathrm{H}_{2}$ and HCN feature, Cernicharo et al. (1999) added an additional layer of $1700 \mathrm{~K}$ between $1 \ldots 3 R_{\star}$ to the adopted model of Keady \& Hinkle (1988) for IRC +10216 . The derived $\mathrm{C}_{2} \mathrm{H}_{2}$ and $\mathrm{HCN}$ abundances in this layer are $2.510^{-5}$ and $1.510^{-5}$, respectively and compare very well with those resulting from our models.

Yamamura et al. (1999a) suggest that the weakening of the $\mathrm{C}_{2} \mathrm{H}_{2}$ absorption at $13.7 \mu \mathrm{m}$ originates from a region around $3 R_{0}$. This radial distance has been estimated by comparing temperatures derived from the observations with time-dependent models of Höfner \& Dorfi (1997). The good agreement with our result is not surprising, since the models of the Vienna group are very similar to ours (cf. Höfner et al. 1996; Woitke 1998). 
Table 4. Percental fraction of the column densities $\left\langle\mathcal{N}_{y}^{\text {col }}\right\rangle$ (see Table 2) in the outer layers of the total column densities

\begin{tabular}{|l||r||r||r||r||r||r|}
\hline & $\mathrm{A}$ & $\mathrm{B}$ & $\mathrm{C}$ & $\mathrm{D}$ & $\mathrm{E}$ & $\mathrm{F}$ \\
\hline \hline $\mathrm{CH}$ & $2 \%$ & $0.5 \%$ & $0.02 \%$ & $0.3 \%$ & $0.02 \%$ & $0.4 \%$ \\
\hline $\mathrm{CN}$ & $5 \%$ & $16 \%$ & $0.1 \%$ & $0.9 \%$ & $0.1 \%$ & $3 \%$ \\
\hline $\mathrm{C}_{2} \mathrm{H}_{2}$ & $100 \%$ & $100 \%$ & $30 \%$ & $61 \%$ & $28 \%$ & $22 \%$ \\
\hline $\mathrm{HCN}$ & $100 \%$ & $100 \%$ & $12 \%$ & $33 \%$ & $12 \%$ & $17 \%$ \\
\hline $\mathrm{C}_{2}$ & $42 \%$ & $44 \%$ & $0.05 \%$ & $0.8 \%$ & $0.2 \%$ & $6 \%$ \\
\hline $\mathrm{C}_{3}$ & $100 \%$ & $100 \%$ & $5 \%$ & $22 \%$ & $5 \%$ & $21 \%$ \\
\hline $\mathrm{CO}$ & $0.6 \%$ & $0.6 \%$ & $0.2 \%$ & $0.6 \%$ & $0.15 \%$ & $0.2 \%$ \\
\hline $\mathrm{TiO}$ & $100 \%$ & $100 \%$ & $2 \%$ & $5 \%$ & $1.6 \%$ & $2 \%$ \\
\hline $\mathrm{SiO}$ & $13 \%$ & $29 \%$ & $0.1 \%$ & $0.2 \%$ & $0.05 \%$ & $0.02 \%$ \\
\hline $\mathrm{H}_{2} \mathrm{O}$ & $0.5 \%$ & $0.002 \%$ & $0.002 \%$ & $0.009 \%$ & $0.001 \%$ & $0.0008 \%$ \\
\hline $\mathrm{CS}$ & $39 \%$ & $76 \%$ & $3.6 \%$ & $11 \%$ & $3 \%$ & $8 \%$ \\
\hline $\mathrm{OH}$ & $810^{-6} \%$ & $210^{-5} \%$ & $610^{-7} \%$ & $210^{-5} \%$ & $510^{-7} \%$ & $110^{-6} \%$ \\
\hline \hline
\end{tabular}

The comparison between the observed and the calculated $\mathrm{C}_{2} \mathrm{H}_{2}$ column densities $\mathcal{N}_{\mathrm{C}_{2} \mathrm{H}_{2}}^{\mathrm{col}}$ shows the best agreement for models with large gas opacity (Planck models). This suggests that dynamic models where the gas opacity has been approximated by Rosseland means or a small constant value produce gas densities which are too high and thereby overestimate the amount of molecules present in the gas phase. Similar conclusions have been drawn by Loidl et al. (1999) who found unrealistically saturated molecular features in the near-infrared spectral region when using models computed with a Rosseland mean or some (small) constant gas opacity value.

A comparison between the models producing the largest amount of dust (constant gas opacity: C, D, E, F) and those containing the smallest amount of dust (Planck models: A, B) shows, that a large dust-to-gas ratio is correlated with large column densities of the molecules. This very general conclusion only holds for models with different gas opacities and is simply related to the considerably higher overall gas densities. A more detailed study among the models calculated with e.g., the Planck mean gas opacity discloses a more differentiated picture: a larger amount of dust is not generally accompanied by a larger column density for all the molecules considered. The only case where all molecular column densities are increased if the dust-to-gas ratio increases is Model D. It is interesting to note, that $\mathrm{C}_{2} \mathrm{H}_{2}, \mathrm{HCN}$, and $\mathrm{H}_{2} \mathrm{O}$ are the only molecules for which the column density increases in all models if the amount of dust increases.

First investigations of time-dependent models for dust forming LPVs with LMC metallicity (Helling et al. 2000a) show that also for such metal-deficient stars the existence of regions with enhanced molecular densities has to be expected. The influence of the dust on the formation of molecular layers is however much smaller in these models, since less dust is formed due to the reduced metal content of the gas. Therefore, also the resulting molecular column densities are by order of magnitudes smaller than in the solar metallicity models. The formation of zones of enhanced molecular densities in the LMC star models is comparable with the oxygen-rich case presented in Woitke et al. (1999) since the pulsation of the star in both cases is the major formation mechanism of these zones.

Jørgensen et al. (2000) suggested a clumpy dust structure in order to explain the flux excess long-ward of $10 \mu \mathrm{m}$ observed simultaneously with a very weak $14 \mu \mathrm{m}$ feature attributed to $\mathrm{C}_{2} \mathrm{H}_{2}$ and $\mathrm{HCN}$ in three carbon stars (TX Psc, V460 Cyg, TT Cyg). This suggestion cannot be supported nor excluded on the basis of our one-dimensional, spherically symmetric models, but multidimensional simulations would be required. A first theoretical approach in this direction has been presented by Woitke et al. (2000), who propose a thermal/radiative instability in dust forming media as physical cause for structure formation processes in the winds of red giants, possibly leading to the formation of dust clouds.

We have not applied a non-LTE treatment of the chemistry in the models presented here, since a complete and consistent determination of the non-equilibrium molecular composition is still too time consuming in the frame work of a time-dependent hydrodynamic modeling. NonLTE chemical abundances in pulsating situations have been calculated by, e.g. Willacy \& Cherchneff (1998) and Duari et al. (1999) for single selected Lagrangian mass elements representing quasi-ballistic trajectories. A comparable non-equilibrium consideration of molecular number densities has been performed by Patzer et al. (1999) for selected Lagrangian mass elements of Model B. Depending on the initial radial distance from the star, these mass elements' trajectories change from a ballistic to a nonballistic behavior. It was demonstrated, that the interaction among the chemical species can become weak already at moderate distances from the star and that the chemical reactions may occur too slowly to alter the abundance 
before the gas is dynamically diluted ("frozen chemistry"). Therefore, the intention of this paper is not to present the ultimate molecular abundances but rather to investigate the influence of the physical processes arising from a dynamical modeling on the molecular stratification.

\section{Conclusions}

1) The column densities of almost all molecules (CN, $\left.\mathrm{C}_{2} \mathrm{H}_{2}, \mathrm{HCN}, \mathrm{C}_{2}, \mathrm{C}_{3}, \mathrm{CO}, \mathrm{TiO}, \mathrm{SiO}, \mathrm{H}_{2} \mathrm{O}, \mathrm{CS}, \mathrm{OH}\right)$ considered in this study increase in the dynamical models where shock waves levitate the atmospheric gas compared to the densities in the static models. Only $\mathrm{CH}$ seems to be virtually unaffected by the star's pulsation. The influence of the shock waves is similar to the oxygen-rich situation which has been discussed in Woitke et al. (1999). Generally, the effect of shock waves on the molecular column densities is amplified by radiation pressure on dust.

2) The effect of shock waves is primarily to increase the column density of the molecules by lifting the gas into non-photospheric regions at $r>2 R_{0}$. The molecular concentrations attain a layered structure and the column densities depend on the phase of pulsation.

3) Beside the levitation by shock waves, also the back-warming (mainly by dust) influences the amount of molecules present, and a competition between the increased column density due to shock waves and the increased temperatures, tending to reduce the molecular densities, determines the amount of molecules present. The increased gas temperature would be accompanied by an increase of the rotational temperature which would result in a broadening of the vibrational bands.

4) Radiation pressure on molecules may also increase the amount of molecules at small radial positions already in the static models if the gas opacity is large. This occurs in competition with a generally decreased density. In addition, strong molecular absorption might cause an additional back-warming.

Acknowledgements. We thank the referee Dr. R. Loidl for valuable comments. Discussions with Dr. P. Woitke and the careful reading of the manuscript by Dr. T. Le Bertre are greatly acknowledged. This work was supported by the FAZITStiftung, the BMBF (grant 053BT13A 6), and partly by the $D F G$ (grant Se 420/19-1). The calculations were performed on the Cray computers of the Konrad-Zuse-Zentrum für Informationstechnik Berlin. Subsequent data analysis has been performed on the WAP-cluster of the physics department of the TU-Berlin.

\section{References}

Aoki, W., Tsuji, T., \& Ohnaka, K. 1998, A\&A, 340, 222

Aoki, W., Tsuji, T., \& Ohnaka, K. 1999, A\&A, 350, 945

Cernicharo, J., Yamamura, I., González-Alfonso, E., et al. 1999, A\&A, 526, L41

Duari, D., Cherchneff, I., \& Willacy, K. 1999, A\&A, 341, L47

Fleischer, A. J., Gauger, A., \& Sedlmayr, E. 1992, A\&A, 266, 321
Gail, H.-P., \& Sedlmayr, E. 1985, A\&A, 148, 183

Gail, H.-P., \& Sedlmayr, E. 1988, A\&A, 206, 153

Gauger, A., Gail, H.-P., \& Sedlmayr, E. 1990, A\&A, 235, 345

Hashimoto, O. 1995, ApJ, 442, 286

Helling, Ch., Arndt, M., T.U., \& Sedmayr, E. 2000a, AG Abstr. Ser., 17,13

(http://export.physik.tu-berlin.de/Publikationen/ Pub100/index.html)

Helling, Ch., Winters, J. M., \& Sedlmayr, E. 2000b, A\&A, 358, 651

Hinkle, K. H. 1978, ApJ, 220, 210

Hinkle, K., \& Barnes, T. 1979, ApJ, 227, 923

Hinkle, K. H., Hall, D. N. B., \& Ridgway, S. T. 1982, ApJ, 252, 697

Hinkle, K. H., Scharlach, W. W. G., \& Hall, D. N. B. 1984, ApJS, 56, 1

Höfner, S., \& Dorfi, E. A. 1997, A\&A, 319, 648

Höfner, S., Fleischer, A. J., Gauger, A., et al. 1996, A\&A, 314, 204

Höfner, S., Jørgensen, U. G., Loidl, R., \& Aringer, B. 1998, A\&A, 340, 497

Jørgensen, U. G., Hron, J., \& Loidl, R. 2000, A\&A, 356, 253

Justtanont, K., Feuchtgruber, H., de Jong, T., et al. 1998, A\&A, 330, L17

Keady, J. J., \& Hinkle, K. H. 1988, ApJ, 331, 539

Keady, J. J., \& Ridgway, S. T. 1993, ApJ, 406, 199

Keady, J. J., Hall, D. N. B., \& Ridgway, S. T. 1988, ApJ, 326, 832

Loidl, R., Höfner, S., Jørgensen, U. G., \& Aringer, B. 1999, A\&A, 342, 531

Matsuura, M., Yamamura, I., Murakami, H., Freund, M. M., \& Tanaka, M. 1999, A\&A, 348, 579

Patzer, A. B. C., Helling, Ch., Winters, J. M., \& Sedlmayr, E. 1999, AG Abstr. Ser., 15, 107

Preibisch, T., Ossenkopf, V., Yorke, H. W., \& Henning, T. 1993, A\&A, 279, 577

Ryde, N., Eriksson, K., \& Gustafsson, B. 1999, A\&A, 341, 579

Tsuji, T. 1986 , A\&A, 156, 8

Tsuji, T. 1988, A\&A, 197, 185

Tsuji, T., Ohnaka, K., Aoki, W., \& Yamamura, I. 1997, A\&A, 320, L1

Unno, W., \& Kondo, M. 1976, PASJ, 28, 347

Unno, W., \& Kondo, M. 1977, PASJ, 29, 693

Willacy, K., \& Cherchneff, I. 1998, A\&A, 330, 676

Winters, J. M., Fleischer, A. J., Le Bertre, T., \& Sedlmayr, E. 1997, A\&A, 326, 305

Winters, J. M., Keady, J. J., Gauger, A., \& Sada, P. V. 2000, A\&A, 359, 651

Woitke, P. 1998, in Cyclical variability in stellar winds, ed. L. Kaper, \& A. W. Fullerton (Springer), 278

Woitke, P., Helling, Ch., Winters, J. M., \& Jeong, K. S. 1999, A\&A, 348, L17

Woitke, P., Sedlmayr, E., \& Lopez, B. 2000, A\&A, 358, 665

Yamamura, I., de Jong, T., Justtanont, K., \& Cami, J. 1998, Ap\&SS, 255, 351

Yamamura, I., de Jong, T., Waters, L. B. F. M., Cami, J., \& Justtanont, K. 1999a, in IAU Symp. 191: AGB stars, ed. T. Le Bertre, A. Lèbre, \& C. Waelkens, 267

Yamamura, I., de Jong, T., Onaka, T., Cami, J., \& Waters, L. B. F. M. 1999b, A\&A, 341, L9 